\title{
Les archives photographiques de la guerre des années 1990. Une approche par l'ethnographie
}

Awel Haouati

\section{OpenEdition}

\section{Journals}

Édition électronique

URL : https://journals.openedition.org/coma/5539

DOI : 10.4000/coma.5539

ISSN : 2275-1742

Éditeur

Institut des textes \& manuscrits modernes (ITEM)

\section{Référence électronique}

Awel Haouati, «Les archives photographiques de la guerre des années 1990. Une approche par l'ethnographie », Continents manuscrits [En ligne], 14 | 2020, mis en ligne le 15 avril 2020, consulté le 12 janvier 2023. URL : http://journals.openedition.org/coma/5539; DOI : https://doi.org/10.4000/coma. 5539

Ce document a été généré automatiquement le 12 janvier 2023.

\section{(c) (1) $(9)$}

Creative Commons - Attribution - Pas d'Utilisation Commerciale - Pas de Modification 4.0 International - CC BY-NC-ND 4.0

https://creativecommons.org/licenses/by-nc-nd/4.0/ 


\title{
Les archives photographiques de la guerre des années 1990. Une approche par l'ethnographie
}

\author{
Awel Haouati
}

1 «L'archive existe, mais elle n'est pas palpable. Je ne peux pas t'en donner l'accès, je ne peux pas te les montrer. ${ }^{1}$ " Les mots sont ceux d'une employée dans un quotidien national algérien, qui m'explique que les archives du journal sont actuellement inaccessibles, y compris pour le personnel. Suite à l'interruption d'un déménagement dans un nouveau bâtiment, pour des raisons politiques et juridiques, seule une partie de ce qui avait été déplacé a pu retourner dans les anciens locaux du journal, les seuls occupés à ce jour. Si le matériel informatique a pu être ramené, les archives, elles, sont restées dans ce nouveau bâtiment, jamais inauguré, et depuis sous scellés. Les photographies que je cherchais à consulter y seraient non seulement bloquées mais désorganisées, de sorte qu'il soit impossible de les consulter. L'affaire est en suspens depuis près de trois ans.

2 Cette scène a eu lieu à Alger, lors d'une enquête en cours que je mène dans le cadre de mes recherches doctorales sur la production photographique dans le contexte du conflit des années $1990^{2}$ en Algérie. Après deux années principalement passées à faire des entretiens avec différents acteurs de la presse et du photojournalisme, j'ai voulu entamer un travail sur les photographies elles-mêmes, en cherchant à dépouiller des journaux et à consulter ce que je désigne moi-même comme des "archives" photographiques. La plupart du temps, les photographes, journalistes et documentalistes avec lesquels je travaille emploient les mots "archive» et, plus couramment, " les archives ${ }^{3}$ » lorsqu'ils se réfèrent à des photographies conservées au sein d'une institution ou d'un journal. Les archives désignent donc l'ensemble des photographies, mais aussi des documents, dossiers de presse et reliures de journaux, rassemblés, constitués en collection et conservés par le journal dans un lieu dédié à cela (une pièce, une cave, une armoire, des boîtes d'archives). Le mot " archives » est plus rarement employé par les photographes lorsqu'ils désignent leurs propres images, 
conservées à leur domicile, dans un espace privé. Celles-ci sont simplement désignées comme des photographies (photos, tsawer en dardja, parler algérien), des photographies de la période des années 1990 ou du «terrorisme ». Pour désigner ce que je cherchais à consulter, j'ai souvent introduit le mot « archives » dans des discussions avec les enquêtés, lesquels le reprenaient par la suite. Afin de simplifier la lecture et éviter les répétitions, les mots " archives » et « archives photographiques » désigneront dans ce texte des photographies produites dans le contexte du conflit des années 1990, qui ne font plus l'objet d'un usage courant et qui constituent un ensemble, plus ou moins ordonné, conservé par des particuliers ou des journaux. Leur consultation n'a cependant pas toujours été évidente pendant mes recherches, les conditions d'accès étant spécifiques à chaque situation et pouvant évoluer au fil du temps. La visibilité de ces photographies, leur accessibilité, dépend en effet du contexte dans lequel elles existent et des personnes qui les conservent, les manipulent - dans le sens tenir dans ses mains -, les font circuler. Le plus souvent, ces archives épousent les contours de la trajectoire, de la vie de celles et ceux qui les détiennent.

3 Afin de mieux comprendre dans quelles conditions ont été réalisées ces photographies, j'ai mené des entretiens avec des personnes qui ont, d'une façon ou d'une autre, participé à leur fabrication et/ou à leur diffusion : photographes, journalistes, éditeurs, rédacteurs en chef, directeurs de publication, documentalistes ${ }^{4}$. Ces discussions étaient une occasion de revenir sur leurs trajectoires et leurs pratiques dans les années 1990, ainsi que sur leur rapport actuel à cette période et aux images de cette guerre. L'objectif était, entre autres, d'essayer de reconstituer une chaîne de fabrication allant du moment de la prise de vue par le photographe à la version imprimée de l'image dans un journal ou diffusée sous une autre forme. Si les entretiens et les observations sur le terrain constituent le matériau principal de cette recherche, le problème de savoir quelle place y occupent les photographies, en tant qu'objets matériels, s'est posé dès le début. Face à des archives photographiques lacunaires, fragmentaires, parfois difficilement accessibles, j'ai été amenée à m'interroger sur la méthode à adopter pour les appréhender. D'une part, parce qu'il ne s'agit pas de les considérer comme de simples sources visuelles sur la période des années 1990, et de réaliser ainsi ce qui a pu être désigné comme une histoire de la photographie ou par la photographie ${ }^{5}$. Cette dernière consisterait en effet en une étude qui se baserait principalement sur les photographies, alors que ce sont davantage les récits des acteurs, leurs pratiques, ainsi que l'ensemble des interactions existant autour des photographies du conflit, qui sont au cœur de mes recherches. D'autre part, parce qu'il existe une réelle difficulté à accéder à certains pans de la production photographique de cette période. Il est, notamment, ardu d'effectuer une "archéologie du document photographique", consistant à remonter aux négatifs des images ou aux tirages de première génération pour en reconstituer la " valeur documentaire originelle $»^{6}$.

Cet article propose certaines pistes d'une réflexion en cours pour une approche des archives photographiques par l'ethnographie, dans le contexte algérien ${ }^{7}$. L'enquête ethnographique, encore en cours, a jusqu'ici consisté en une série d'entretiens, semidirectifs, approfondis et répétés au fil des mois, avec une cinquantaine de personnes, en Algérie principalement, mais aussi en France. Ces entretiens, sur le temps long et qui nécessitent de nouer une relation de confiance avec mes interlocuteurs, ont été complétés par un dépouillement de titres de presse algériens, ainsi que par la consultation d'archives photographiques, lorsque cela a été possible. Je me pencherai ici sur le cas des images réalisées par des photographes de presse algériens dans les 
années 1990, diffusées dans la presse nationale. Celles-ci sont appréhendées comme des objets photographiques, matériels, façonnés par divers contextes et acteurs, à des moments différents. Deux temps rythment la structure de l'article : celui des années 1990 et celui de l'enquête, réalisée entre 2017 et 2020. Le temps des années 1990 est celui dans lequel ont été fabriquées et diffusées ces photographies. Il désigne à la fois un contexte historique et politique, et un environnement social et professionnel, celui de la presse algérienne à cette période. Temps de crise, il est marqué par l'urgence, la violence et l'instabilité. Ce contexte, de même que les pratiques des acteurs de la presse à cette période, ont participé à façonner les archives, leur donnant la forme sous laquelle elle se présentent aujourd'hui. Le temps de l'enquête, quant à lui, est celui du retour sur le passé effectué par les acteurs au moment de l'entretien. Il est aussi celui de l'exhumation de ces photographies dans le présent, par différents acteurs. En partant de la notion de "vie sociale» des objets et des images ${ }^{8}$, l'article retrace la trajectoire de photographies de presse, entre la période à laquelle elles ont été produites, dans les années 1990, et le temps présent. Diffusées à l'origine comme des images rattachées à une actualité, celle de la guerre et de la violence, elles rejoignent aujourd'hui d'autres régimes d'existence, de circulations et de conservation. Je mobiliserai en ce sens les réflexions de l'artiste libanais Akram Zaatari ${ }^{9}$ autour de la vie des archives photographiques et leur passage par différents régimes d'existence au cours du temps. Cette approche permettra également d'interroger le rôle que joue le chercheur - acteur parmi les acteurs qui manipulent les images - qui, dans sa quête d'archives pour nourrir son projet, peut intervenir, de façon spontanée ou consciente, sur la vie de ces images et en modifier la trajectoire. Il s'agira enfin de présenter une ébauche de cartographie, non seulement des archives photographiques de la "décennie »1990, mais des pratiques liées à ces archives, à la fois dans l'espace des rédactions de journaux et dans l'espace privé, domestique.

\section{La photographie de presse dans le contexte des années 1990 : une pratique de l'urgence}

5 La brève ouverture démocratique qui a lieu en Algérie à partir de la réforme de la Constitution en 1989, consacrant la fin du parti unique et l'autorisation des " associations à caractère politique » (les partis), se fait simultanément à une ouverture du champ de la presse. En mars 1990, une circulaire rédigée par le gouvernement de Mouloud Hamrouche, premier ministre, suivie par la promulgation du code de l'information en avril de la même année, encourage les partis nouvellement créés et les journalistes voulant quitter la presse gouvernementale à lancer leurs propres publications. Trois années de salaire sont ainsi octroyées aux anciens journalistes de la presse publique voulant se lancer dans la presse privée ${ }^{10}$. C'est ainsi que sont créés les premiers journaux privés comme El-Watan, Le Soir d'Algérie ou encore El-Khabar, à partir d'équipes majoritairement issues de la presse étatique, notamment du journal ElMoudjahid, alors organe du parti du FLN (Front de libération nationale).

6 De nombreuses personnes se forment alors au journalisme. Souvent décrit par mes interlocuteurs comme une brèche historique et politique, c'est un moment d'exaltation et d'expérimentation dans le milieu de la presse. Certains journaux, s'inspirant de revues publiques comme Révolution africaine, organe central du FLN, ou de publications françaises comme Libération, accordent une importance non négligeable à l'image et au 
graphisme. Des photographes déjà actifs au sein de la presse gouvernementale passent dans la presse privée, alors qu'une nouvelle génération se forme sur le tas au métier de photographe de presse. S'il existe une formation en journalisme à cette période, ce n'est pas le cas pour les métiers visuels de la presse écrite, en dehors d'une formation de courte durée au CNDPI (Centre national de documentation de presse d'images et d'information) à Alger et de stages de photographie proposés par les Maisons de jeunes. La plupart des photographes rencontrés ont appris le métier en le pratiquant lorsqu'ils ont rejoint une rédaction. En janvier 1992, l'arrêt du processus électoral des législatives, après la victoire du Front islamique du salut (FIS ${ }^{11}$ et la démission du président Chadli Bendjedid, précipite progressivement le pays dans la violence. Plusieurs groupes s'affrontent alors, d'une part, l'armée islamique du salut (AIS) et les groupes islamiques armés (GIA), d'autre part l'armée nationale populaire, ainsi que différentes forces de sécurité et milices civiles. La pratique de la photographie de presse se développe dans ce contexte politique, fortement polarisé par la violence.

7 La couverture de la guerre, qui n'est pas désignée comme telle, se fait parallèlement à celle des événements culturels, politiques et des reportages dits de société. Mais c'est « l'actualité » ou « le news »- pour reprendre le mot d'une de mes interlocutrices - qui prend une place prépondérante dans la presse. Si le fonctionnement du traitement de l'actualité par la presse quotidienne est comparable à d'autres contextes, la spécificité de l'Algérie des années 1990 se situe dans le rythme auquel s'enchaînent les événements. Les faits ou les actes de violence - attentats, assassinats et, plus tard, massacres - se succèdent à un rythme quasi quotidien, et il est possible d'en suivre la trame en feuilletant les journaux année après année. Dans les récits oraux, les dates et les années se confondent, et accentuent cette impression d'un temps compressé, rapide. 


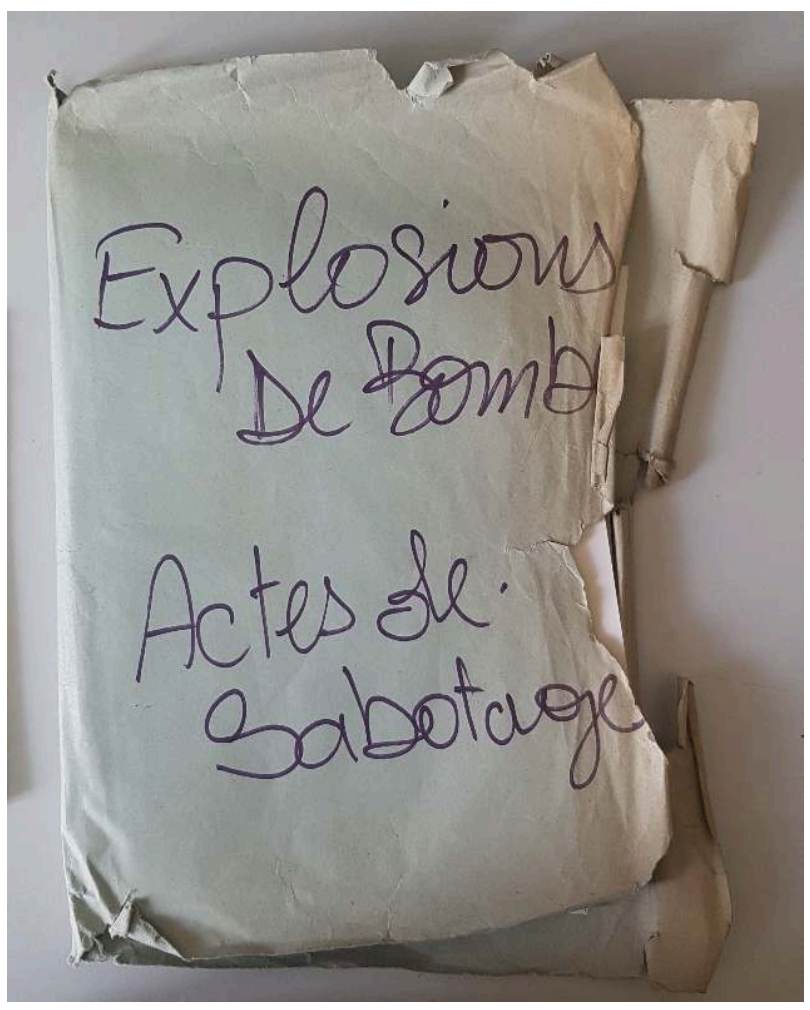

Chemise contenant les tirages photographiques classés dans la thématique «Explosions de bombe. Actes de sabotage » dans les archives d'un quotidien public algérien francophone.

Photographie de l'auteure, Alger, avril 2019.

De la même façon que le chroniqueur et auteur Sid-Ahmed Semiane parle d'« écriture de l'urgence » en évoquant le contexte dans lequel il a rédigé les articles réunis dans son recueil paru en $2005^{12}$, il semble juste de désigner l'ensemble des pratiques liées à la photographie de presse dans le contexte du conflit des années 1990 comme des pratiques de l'urgence. Cette urgence est décelable dans les récits des acteurs évoquant la rapidité avec laquelle les événements se déroulent entre le moment où une information est transmise aux équipes du journal et celui où sont dépêchés, sur les lieux d'un attentat ou d'un massacre, les journalistes et les photographes. Ces derniers arrivent en effet après l'événement, souvent en même temps que les autorités (forces de sécurité, gendarmerie, police scientifique) et les pompiers. Il faut alors travailler rapidement pour ne pas se faire remarquer, recueillir les informations, essayer de compter les victimes et inscrire leurs noms quand cela est possible, faire des photographies à la hâte, des réticences et des interdictions pouvant provenir des différents acteurs présents sur place. Cette urgence se fait aussi ressentir dans les récits relatant le retour dans les rédactions après la couverture d'un événement, lorsque les photographes doivent développer leurs films rapidement pour proposer une sélection de tirages à la rédaction chargée de choisir une image à publier dès le lendemain.

Si les actes de violence, quotidiens et survenant en différents points du territoire national, sont trop nombreux pour faire l'objet d'une couverture journalistique systématique, la sélection des événements à couvrir et à mettre en avant est réalisée par les équipes de rédaction en fonction de leurs lignes éditoriales, de leur marge de manœuvre dans le champ politique de cette période et de différentes formes de 
censures. La couverture de ces événements par les photographes se fait elle aussi de manière ponctuelle. En feuilletant par exemple les journaux Liberté, El-Watan et Horizons $^{13}$, entre 1990 et 1999, l'on s'aperçoit que les faits de violence font rarement l'objet d'une Une. La plupart de ces informations sont publiées à l'intérieur ou en dernière page, dans un encadré qui porte tour à tour les titres : «incidents ", «ordre public ", «terrorisme » ou encore " lutte antiterroriste ». Le nom de ces rubriques peut changer au fil des mois et des années, parfois au sein d'une même publication. Souvent les textes sont brefs, télégraphiques, et ne sont accompagnés d'aucune photographie. Les articles plus longs et publiés avec une photographie concernent des faits mis en avant dans les journaux comme des événements plus importants que les autres. Ces derniers sont dans certains cas publiés en Une, avec un gros titre et une photographie «éclatée» occupant la moitié ou la presque totalité de la page. Une telle hiérarchisation des faits dépend de différents paramètres et enjeux, aussi bien pratiques et éditoriaux que factuels et politiques, qui ne seront pas développés dans cet article. Dans ce contexte de violence, d'instabilité et d'urgence, les photographies du conflit sont diffusées par la presse quotidienne comme des images d'actualité. Rattachées à des événements précis, elles ont une "valeur d'information $»^{14}$ dans la mesure où elles sont diffusées pour accompagner, appuyer ou simplement illustrer des articles portant sur ces événements. Lorsqu'un événement n'a pas fait l'objet d'une couverture photographique, les équipes de la documentation ont la possibilité de puiser dans leurs archives, ou dans celles d'autres organes et agences de presse nationales ou internationales, pour proposer à la rédaction les images d'un événement similaire, survenu plus tôt. Dans ce cas de figure, les images sont détachées des événements auxquels elles se réfèrent à l'origine, pour être rattachées à des thématiques plus larges, ce qui permet de les diffuser à nouveau. Le fait d'être classées en thématiques et archivées, pour potentiellement être réutilisées, fait passer ces images d'une temporalité quotidienne à une temporalité plus étendue. Elles demeurent des images d'actualité exploitables pour la presse, tant que le contexte reste plus ou moins le même, celui de la violence, de la guerre.

\section{«On n'utilise plus ces photos, donc on les a oubliées. »}

$10 \mathrm{Au}$ cours de mes recherches, j'ai pu consulter des archives photographiques au sein de quotidiens nationaux algériens. Dans les boîtes d'archives où sont conservés les tirages ${ }^{15}$ des années 1990, le classement et les thématiques qui avaient été mis en place par les documentalistes à cette période sont plus ou moins restés les mêmes. Des motsclés inscrits sur la cote de ces boîtes indiquent les catégories suivantes : " terrorisme ", « massacres », «assassinats ", " actes de sabotage », "armée ", " police ", " patriotes $»^{16}$. À l'intérieur de ces boîtes, chaque événement est classé dans un sous-dossier portant une légende indiquant la date et souvent le lieu de l'événement. Par exemple « Massacre de Oued el Alleug. Blida (13.11.96) ». Le matériel utilisé pour ces sous-dossiers se résume à des chemises en papier de différentes couleurs ou des enveloppes de courrier recyclées pour cet usage. Ce détail peut indiquer l'urgence dans laquelle a été réalisé le tri et le classement de ces tirages, comme il peut être le signe d'un manque de moyens investis dans l'archivage des photographies par les journaux, dans les années 1990 mais aussi aujourd'hui. Pendant 
que je consulte les images conservées dans l'une des deux rédactions, une documentaliste me dit : " On n'utilise plus ces photos, donc on les a oubliées ${ }^{17}$. " J'avais alors passé plusieurs jours dans son bureau, dans l'entresol du bâtiment, et l'avais sollicitée pour aller chercher les journaux (ces derniers se présentent souvent sous la forme de reliures compilant deux ou trois mois) et quelques boites de photographies accessibles. La pièce où sont rangées les archives - plusieurs dizaines de boîtes - est sombre et humide. L'aération ne fonctionne pas très bien et la poussière a recouvert les boîtes et les reliures. La documentaliste m'explique qu' «ils » - la direction du journal n'ont jamais investi dans du matériel pour conserver ces archives dans de bonnes conditions. Une photographe dans le même journal m'avait parlé de ces archives quelques mois plus tôt dans ces mots : « Toi tu veux faire une thèse, tu viens au journal, tu demandes les archives. Si tu voyais dans quel état elles sont, les archives... Tu en pleurerais ${ }^{18}$. $\gg$ Ma présence dans les locaux du journal relance la discussion sur les archives entre les travailleurs, sur l'état de conservation des photographies et les moyens fournis par la direction. La photographe avait lancé un projet de numérisation quelques années auparavant, mais l'équipe qui s'en chargeait avait reproduit les tirages et les négatifs en très basse définition; ils étaient inexploitables. Dans le bureau du directeur de publication, elle me présente, lui parle de mon souhait de consulter les archives photographiques et de la possibilité de relancer le projet de numérisation. Le directeur la soutient mais ajoute que le journal peine à sortir et ne peut se permettre d'investir de l'argent dans ce genre de projet. Dans un autre journal, les boîtes contenant les photographies des « années noires » et du «terrorisme » sont conservées dans la cave du bâtiment, avec toutes les archives du journal. L'humidité et l'odeur de moisissure y sont si fortes que le personnel de la documentation lui-même est réticent à l'idée de s'y rendre pour me faire remonter quelques boîtes à consulter. 
Figure 2

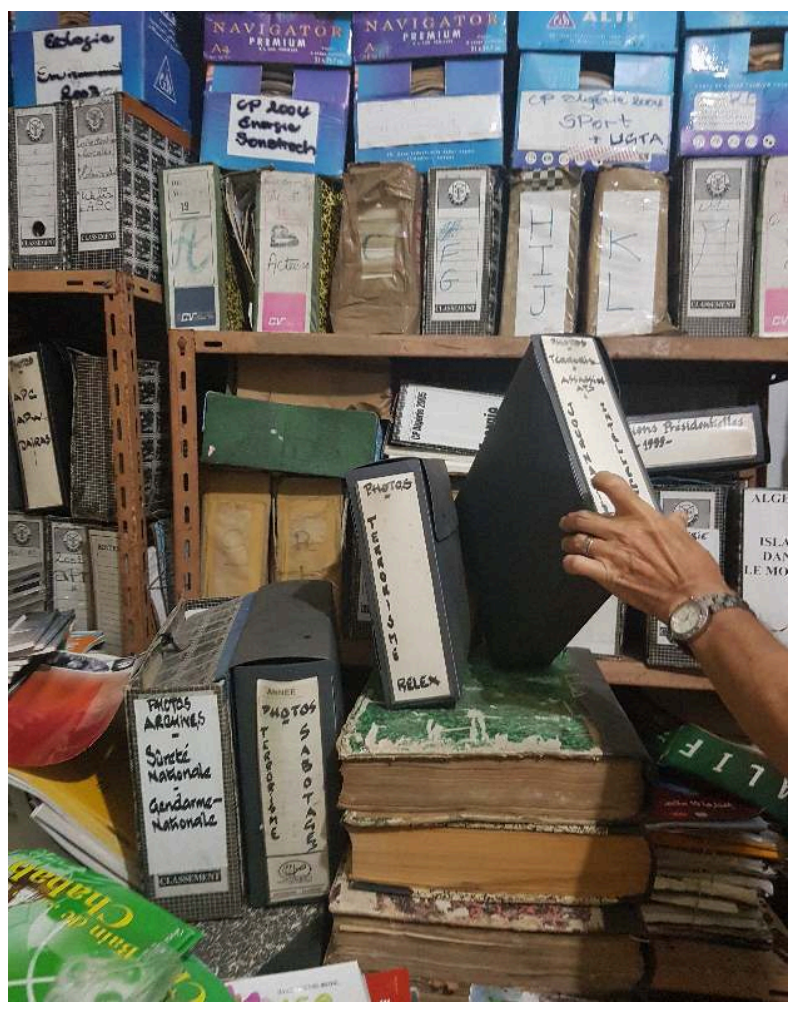

Le documentaliste d'un quotidien public algérien lors d'une brève visite dans les sous-sols du bâtiment où sont conservées les archives, notamment photographiques, du journal.

Photographie de l'auteure, Alger, juin 2019

Les photographies du conflit des années 1990 cessent d'être des images d'actualité à partir du moment où l'Algérie sort progressivement de la violence, au début des années 2000. Les attentats et les massacres se font plus rares, sans s'arrêter complètement dans les années 2000. La politique de lutte antiterroriste de l'État algérien et de son armée n'est plus autant mise en avant dans les médias, cédant la place à une politique de "réconciliation » et d'amnistie mise en place par le président Abdelaziz Bouteflika à son arrivée au pouvoir ${ }^{19}$. Pour la presse, ces images basculent dans un statut obsolète qui poussent la plupart des journaux encore existants à les reléguer dans des armoires, des caves, d'où elles ne sont quasiment jamais extirpées. Plusieurs facteurs peuvent expliquer le fait que ces photographies n'ont plus circulé audelà de la limite temporelle de l'actualité à laquelle elles se rattachent. D'abord, le fait que les journaux ne considèrent pas comme une priorité la question de la préservation et de la numérisation de leurs archives photographiques et, par conséquent, n'investissent pas dans des structures et du matériel qui leur permettrait non seulement de conserver ces images mais aussi de les exploiter. Ensuite, la rareté de canaux de diffusion et de supports à travers lesquels ces images pourraient circuler : publications, revues spécialisées, magazines, expositions. Si quelques publications existent sur les années 1990, elles ne mobilisent généralement pas d'images; cette période reste peu investie, notamment par la recherche. Les photographies de cette période ne sont quasiment jamais exhumées, sinon par des artistes et des cinéastes ${ }^{20}$. Enfin, on peut se demander dans quelle mesure les images des années 1990 ont leur place dans le contexte actuel, à quel moment elles sont les plus à même de réapparaître et sous quelles formes. La remise en circulation de photographies du conflit soulève en 
effet plusieurs problèmes, pour leurs auteurs, mais aussi pour les publics qui pourraient les recevoir et les acteurs qui cherchent à les exhumer et les montrer : photographes, chercheurs, artistes, héritiers, éditeurs. Comment les diffuser dans le contexte actuel, qu'est-il possible de montrer et où? Faut-il attendre le moment opportun pour les diffuser et comment définir ce moment? Sont-elles diffusées comme des documents historiques ou des œuvres d'art?

\section{Des archives privées en fragments, reflets d'une instabilité du quotidien pendant la guerre}

Dans le contexte d'instabilité et d'urgence des années 1990, toutes les photographies réalisées pour la presse ne sont pas systématiquement conservées par les journaux. Plusieurs photographes ont fait le choix, à cette période, de garder leurs négatifs, pour ne remettre aux rédactions et à la documentation des journaux que des tirages. Ainsi, Ammar Bouras, artiste plasticien et photographe de presse dans les années 1990, explique : «À Alger Républicain [...]. J'ai conclu un accord, avec l'administration, quand je suis arrivé : je garde mes négatifs. C'est-à-dire que les négatifs, c'est les miens, coûte que coûte, je ne peux pas les laisser, je ne peux pas. C'est à moi ${ }^{21}$. » Bouras est donc resté propriétaire de ses clichés, tandis que le journal au sein duquel il travaillait pouvait diffuser les images issues des tirages qu'il remettait. À cette période (comme aujourd'hui), la question de la propriété et des droits de l'image n'est pas rigoureusement établie dans la presse algérienne, aussi bien d'un point de vue légal que dans les pratiques. Des photographes ont ainsi évoqué leurs «bagarres » quotidiennes au sein des rédactions pour ajouter leur signature aux photographies publiées dans le journal. C'est aussi un moment où les journalistes et les photographes changent souvent de poste, "papillonnent " (selon le terme d'une interlocutrice) de journal en journal, pour différentes raisons. Dans un contexte de multiplication des titres et d'expérimentation, on pouvait être à la recherche de meilleures conditions de travail et d'un meilleur salaire, mais aussi aller vers un titre dont la ligne éditoriale était plus souple ou correspondait davantage à des positions politiques personnelles. Subissant d'importantes pressions, notamment de la part du pouvoir algérien, plusieurs journaux ont connu des épisodes de suspension temporaires ou définitifs, conduisant des équipes entières à se répartir entre les rédactions subsistantes. L'incertitude quant à l'utilisation des images par les rédactions, ajoutée à ces différentes formes d'instabilité et au développement du travail en freelance, a ainsi joué un rôle dans le choix de certains photographes de garder leurs clichés chez eux. De plus, le fait que les journaux accordent peu d'importance, voire négligent parfois leurs archives, et n'allouent pas de moyens à leur conservation, amène les photographes à vouloir garder leurs archives dans des endroits plus sûrs.

$13 \mathrm{Au}$ cours de mes recherches, parallèlement au travail sur les fonds photographiques conservés dans les journaux, j'ai ainsi pu consulter les archives privées d'une demidouzaine de photographes, conservées à leur domicile, sur leur lieu de travail dans un espace privatif (une armoire, un bureau) ou dans un atelier. L'accès, même partiel, à ces archives dans la sphère privée permet non seulement de voir les photographies mais aussi d'étudier un ensemble de pratiques ordinaires et domestiques de l'archivage. Certains photographes ont mis en place un système personnalisé de rangement et de classement de leurs propres archives (par thématiques et par années), souvent avec les 
moyens du bord (enveloppes récupérées, feuilles A4 pliées et enroulées autour des négatifs de chaque série, boîtes de papier photo réutilisées pour ranger les tirages). Dans certains cas, une partie ou la totalité de leur production a été numérisée et copiée sur des disques durs ou, quand le travail a été fait plus tôt, sur des CD. L'ensemble de ces photographies a ainsi été organisé de façon systématique par leurs auteurs de sorte à constituer des archives, c'est-à-dire des collections conservées dans le but de durer dans le temps.

14 Mais les cas où les photographies conservées dans l'espace privé se présentent sous la forme d'un ensemble uniforme, complet et à la chronologie continue sont rares. La plupart des photographes n'ont pu me montrer que des fragments de leur travail des années 1990, dont des parties avaient été éparpillées, perdues au cours de leur trajectoire, jamais récupérées auprès de leurs anciens employeurs (agences, journaux) ou de proches. Dans certains cas, des photographies ont été détruites, le plus souvent par accident du fait de mauvaises conditions de conservation (humidité, inondations). Pendant le conflit, nombreuses sont les personnes qui déménagent pour des raisons de sécurité, par précaution pour leur entourage et par peur. Les travailleurs de la presse font alors partie des catégories les plus visées par les assassinats. Les menaces, notamment de la part des branches armées des islamistes, et les rumeurs sont fréquentes, poussant les journalistes à quitter leur domicile familial pour s'installer ailleurs. Certains d'entre eux habitent pendant plusieurs années des chambres dans des «logements sécuritaires » mis en place pour la presse par l'État algérien, notamment dans des hôtels de la banlieue ouest d'Alger ${ }^{22}$. Parmi les photographes qui ont accepté de me montrer leur travail, certains en ont laissé une partie dans la maison de leurs parents, ou dans ces anciennes chambres d'hôtels, et par manque d'espace, ou parce qu'ils n'ont pas eu le temps de s'en occuper, leurs photographies demeurent là où ils les ont les laissées. Rassembler l'ensemble de ces photographies dispersées entre plusieurs journaux, dont certains ont fermé, et en différents lieux, représente en effet un travail colossal qu'il n'est pas aisé d'entreprendre seul. 
Figure 3

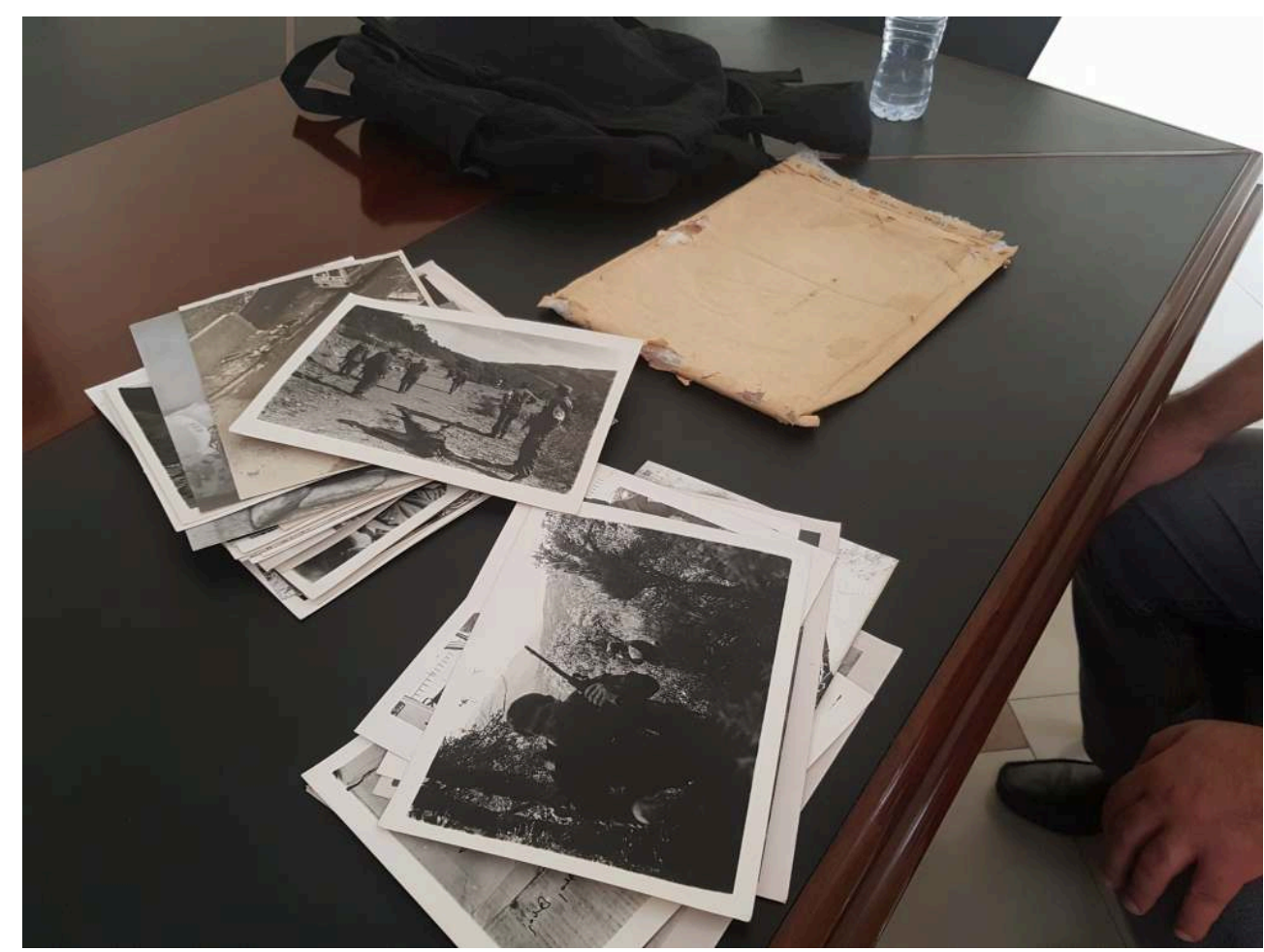

Un photographe de presse apporte les quelques tirages qu'il a gardés chez lui, lors de l'entretien. II affirme que ces photographies sont tout ce qu'il a pu conserver de son travail pendant les années 1990.

Photographie de l'auteure, Alger, octobre 2018.

\section{Les photographies de la « décennie » dans le présent: des images latentes?}

15 À l'exception de quelques expositions organisées en Algérie et en France, faisant la rétrospective de la production photographique algérienne dans un sens large ${ }^{23}$, les occasions de voir ces images resurgir aujourd'hui sont rares. Il arrive que des photographies soient publiées sur les réseaux sociaux, à l'occasion de dates commémoratives d'événements de la "décennie » (attentats, assassinats, massacres). Mais, le plus souvent, il s'agit de photographies diffusées par des agences de presse internationales, donc, accessibles sur Internet ${ }^{24}$. Des images devenues iconiques connaissent aussi une diffusion ponctuelle, comme la célèbre photographie de Hocine Zaourar prise au lendemain du massacre de Bentalha et diffusée en 1997 par l'AFP et largement diffusée depuis plusieurs années, ou le travail du photographe suisse Michael Von Graffenried, circulant sous forme de livres et de catalogues. En dehors de ces quelques exemples, le constat est le même pour une grande partie des photographies de presse du conflit des années 1990 : celles-ci ne circulent plus et ce depuis le début des années 2000. Cette observation nous amène à émettre l'hypothèse que ces photographies sont, pour un temps donné, latentes ${ }^{25}$. Elles peuvent ainsi resurgir dans le présent, à la lumière d'un événement ou d'une conjoncture - politique, historique - spécifique, ou du fait de l'intervention de personnes qui agissent sur leur 
trajectoire, la modifient en remettant ces images en circulation, en les rendant à nouveau visibles.

Dans un entretien avec Anthony Downey, professeur en culture visuelle, l'artiste libanais Akram Zaatari évoque son projet autour du studio Shehrazade, tenu par le photographe Hashem El Madani dans la ville de Saïda, au Liban, qui a été actif entre les années 1950 et les années 1970. Pour Zaatari, si lui-même a pu avoir accès aux archives d'El Madani, les déplacer de leur contexte d'origine et les intégrer à son œuvre, c'est parce que le studio n'était déjà plus fonctionnel à l'époque où il a sollicité le photographe: «[...] si le studio avait été actif aujourd'hui, si l'économie de la photographie avait été active aujourd'hui, Madani ne m'aurait pas laissé prendre un seul de ses négatifs en dehors de son archive. [...] La mort de son économie a donc permis à mon projet d'exister... ${ }^{26}$ » De la même façon que Zaatari parle d'une économie propre au studio d'El Madani, il est possible de désigner le contexte de production et de diffusion des photographies de presse de l'Algérie des années 1990 comme une économie spécifique à une période donnée. Celle-ci se développe tout au long des années 1990 et «meurt » au début des années 2000, faisant basculer l'ensemble de ces images d'actualité dans d'autres formes d'économie, de régimes d'existence à la fois sociaux et politiques.

Parmi ces nouvelles formes d'économie, dans lesquelles les images sont susceptibles de circuler, nous pouvons citer celle du livre et de l'édition. Si plusieurs photographes ont le projet de faire un livre pour regrouper l'ensemble de leur travail, notamment celui des années 1990, la plupart ne l'ont pas encore concrétisé. Le livre du photographe et plasticien Ammar Bouras, 1990-1995. Algérie, chronique photographique est aujourd'hui l'un des seuls qui réunisse des photographies produites à cette période et qui ait été édité en Algérie $^{27}$. La parution de l'ouvrage au début de l'année $2019^{28}$, alors que le pays connaît le plus important soulèvement depuis 1988 et 2001, bénéficie d'une grande couverture médiatique et suscite beaucoup d'intérêt, signe de la rareté de ce genre de projets autour des images des années 1990. Le livre est le fruit d'un travail de numérisation de ses négatifs, que Bouras a mené pendant près de trois ans, et a vu le jour suite à une suggestion de son ami et collègue Adlène Meddi, journaliste et écrivain. Lorsque je rencontre le photographe en novembre 2018, celui-ci m'explique que Meddi lui avait rendu visite à son atelier et l'avait trouvé en train de scanner ses négatifs. Il lui a alors suggéré d'en faire un livre et a proposé le projet à une maison d'édition, qui a accepté. Ainsi, l'intervention de différents acteurs - du photographe au journaliste, en passant par l'équipe de la maison d'édition - participe à modifier la trajectoire des photographies de Ammar Bouras, du moins une sélection issue de son œuvre, et à faire entrer celle-ci dans une nouvelle forme d'existence. Ces images circulent à présent sous la forme d'un beau-livre, acquérant une nouvelle valeur, commerciale, marchande, mais aussi mémorielle et culturelle. Le fait que plusieurs photographes aient le projet de faire un livre à partir de leur travail pendant les années 1990, ainsi que l'entrée de l'Algérie dans une nouvelle séquence historique et politique depuis février 2019, pourrait amener une partie de ces images à circuler davantage dans le présent, sous cette forme ou sous une autre. 
Figure 4

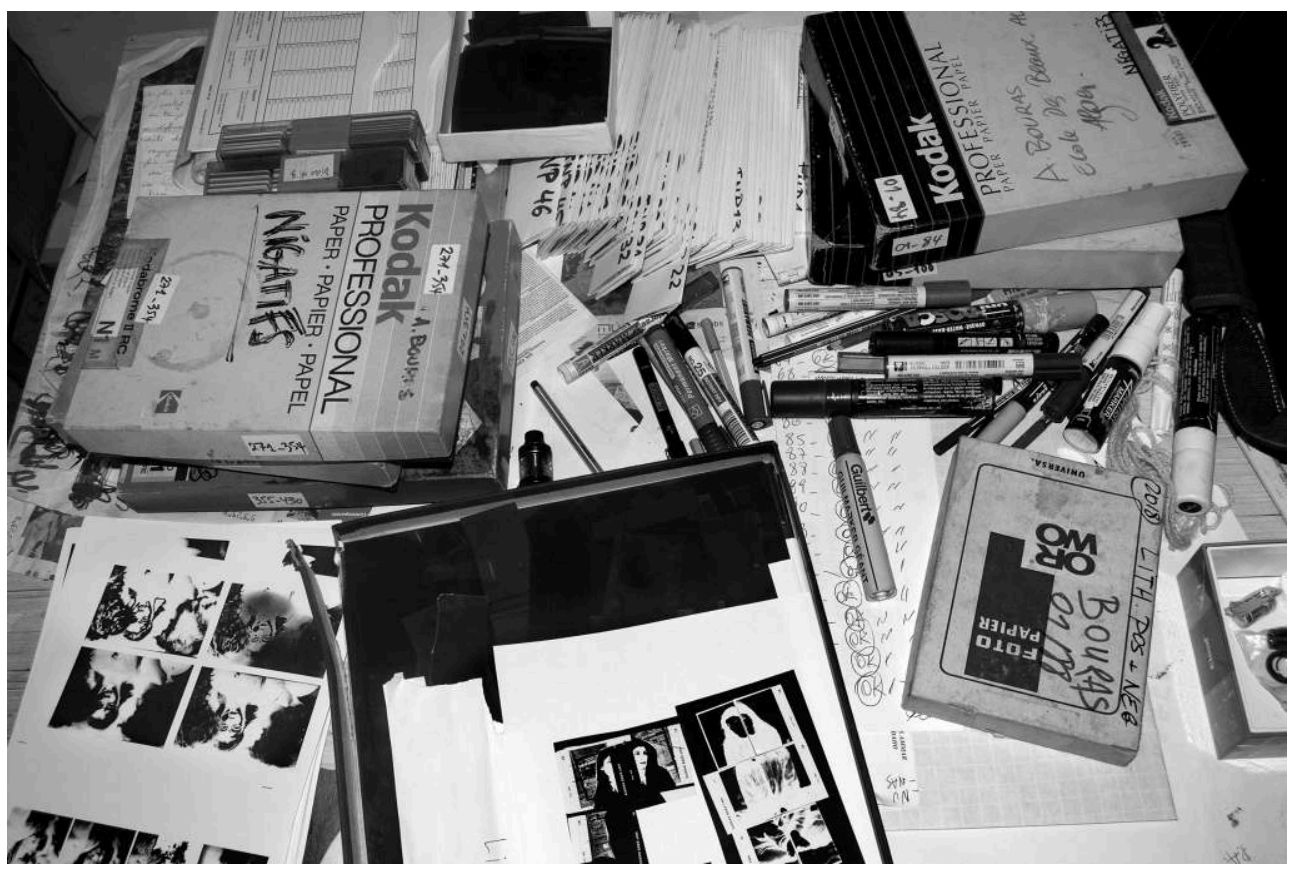

Archives du photographe et plasticien Ammar Bouras.

Photographie d'Ammar Bouras publiée dans son livre 1990-1995. Algérie, chronique photographique, Barzakh, Alger, 2018.

\section{Exhumer, déplacer et catégoriser l'œuvre d'un photographe disparu : la genèse d'une archive?}

Effectuer un travail de recherche n'est pas non plus sans conséquences sur la trajectoire des images et leur « vie sociale ». En cherchant à consulter des fonds au sein des journaux ou chez les photographes, j'amène les différents acteurs avec lesquels je suis en contact à aller chercher dans leurs cartons pour exhumer des objets photographiques qui n'avaient, souvent, pas bougé depuis plusieurs années. Le fait de les pousser à chercher ces photographies, rangées dans des armoires, des sacs et des boîtes, pour les regarder ensemble, en parler, y apposer des récits, est une forme d'intervention dans la vie sociale de ces objets photographiques mais aussi dans celle des personnes qui les conservent.

19 À l'été de l'année 2017, je repris contact avec les enfants d'un photographe de presse algérien, décédé à la fin des années 1990. J'exposai mon sujet de recherche au fils du photographe, qui est un ami d'enfance - appelons-le B. - et de mon intérêt pour le travail et l'histoire de son père. Il se trouva qu'il avait récupéré, peu de temps avant notre échange, une partie de photographies de son père auprès d'un proche, et me proposait de me les montrer. Nous avons donc convenu d'un rendez-vous chez sa sœur. Le soir du rendez-vous, B. ramène les photographies dans une grande valise rouge, plus facile à transporter que les cartons et sachets dans lesquels le proche les lui avait remises. Après le repas, il nous propose de nous mettre "au travail», c'est-à-dire ouvrir la valise, commencer à regarder et trier les images qui se présentent alors sous la forme de tirages, de négatifs classés dans des feuillets transparents et de diapositives. Nous déballons les sacs, ouvrons les boîtes contenant les tirages et en étalons le 
contenu sur le tapis du salon, bientôt recouvert de tirages noir et blanc. L'ensemble est impressionnant, c'est la première fois que je vois des photographies de cette période, de la guerre, de ses lieux et de ses acteurs. Si B. y a déjà jeté un coup d'œil, sans s'y plonger véritablement, sa sœur, elle, découvre les images en même temps que moi. Spontanément, nous constituons différents tas à partir des tirages, selon des thématiques inventées sur place, que nous inscrivons sur des feuilles blanches pliées en deux et dans lesquelles nous glissons les photos: patriotes et chnabet (gardes communaux), militaires, policiers, scènes de vie diverses, attentats, rassemblements et meetings du FIS, manifestations politiques, photos du Sahara, portraits d'hommes politiques... Nous essayons tant bien que mal de pallier au manque d'informations sur les tirages - dont le dos ne porte aucune légende, aucune date - en nous appuyant sur les quelques connaissances que nous avons sur le contexte des années 1990 et les différents acteurs de la scène politique d'alors. Après ce premier rendez-vous, B. m'apporte les photographies à la maison pour continuer « le travail » dans un espace plus grand, où nous pouvons étaler et classer les triages à notre rythme, avant de les ranger. Nous nous renseignons alors sur les techniques d'archivage, le matériel nécessaire, auprès de personnes dont c'est le métier et sur Internet. En près de trois mois, nous avions trié les tirages, isolé ceux qui étaient atteints par la moisissure et classé les autres dans des enveloppes et des boîtes d'archives, en reprenant les thématiques évoquées précédemment. J'ai par la suite entrepris d'en scanner une partie pour les avoir sur mon ordinateur et ainsi les montrer plus facilement pendant mes entretiens, pour avancer dans l'enquête concernant le travail du photographe. À ce jour, l'inventaire de ses archives compte près de sept-cent tirages photographiques, noir et blanc pour la plupart et de différentes dimensions, dont près de deux cents ont été numérisés.

20 À la mort du photographe, son travail a été dispersé entre son domicile et ceux d'amis ou de collègues. Un membre de sa famille, N., a entrepris de récupérer les photographies et de les rassembler. Certaines d'entre elles ont fait l'objet d'expositions en hommage au photographe, organisées en partie par N. Une partie de ces images a, semble-t-il, été vendue ou donnée, peut-être à l'époque où elles avaient encore valeur d'images d'actualité. Nous nous sommes en effet aperçu, avec son fils, en classant les tirages et en croisant les sources (entretiens avec des amis et collègues du photographe, journaux), qu'il manquait tout un pan des tirages, mais surtout des négatifs, de ses reportages. Il est aussi possible que certaines parties aient été abîmées ou détruites, suite aux immenses inondations qui ont touché la capitale en 2003, notamment dans le quartier de Bab-el-oued où se trouvait la maison dans laquelle étaient stockées les photographies. Répondant aux sollicitations du fils du photographe, N. a donc remis à celui-ci la production de son père, du moins ce qu'il en restait, avant que le fils ne me les apporte à son tour à mon domicile. Ces photographies ont donc connu plusieurs itinéraires au cours de ces vingt dernières années. Chacune des personnes qui les a eues entre les mains y est intervenue de différentes façons, chaque contexte traversé a eu un impact sur leur forme globale et leur matérialité. Ces interventions et ces gestes, spontanés et a priori anodins, constituent peut-être le point de départ de la genèse des archives de ce photographe, en tant que collection plus ou moins cohérente de photographies. En ce sens, les notions de "performance», de "déplacement» (displacement) et de «fouille " (excavation, digging), développées par Akram Zaatari, sont utiles pour décrire ce processus par lequel se constituent les archives, entre les mains de différents acteurs. Celles-ci sont appréhendées non pas comme quelque chose de fixe 
et de figé, mais en constante évolution au fil du temps. En déplaçant, en classant et en apposant des catégories sur des objets photographiques, nous avons, avec le fils du photographe, ajouté une " couche » (layer) ${ }^{29}$ d'interprétation et de "vie » à ces images et participé, par nos gestes et nos pratiques ordinaires, quotidiennes - qui désignent ici un ensemble de pratiques et de gestes non professionnels, domestiques ou encore amateurs - à les faire entrer dans un nouveau régime d'existence, celui des archives ou de l'archive. Mon intervention en tant que doctorante, tout comme celle de B. et avant lui N., pourraient donc être appréhendées comme des " performances ", aussi bien dans la vie sociale, économique et politique de ces photographies, que dans celle des personnes elles-mêmes.

21 Un tel cheminement à travers les archives photographiques du conflit algérien des années 1990 amène à interroger la notion "d'images manquantes ", souvent mobilisée pour parler de cette période. Dans certains écrits académiques comme dans les médias, on a en effet souvent désigné ce conflit comme une "guerre sans images», une "guerre invisible ${ }^{30}$. Une telle affirmation parait excessive, même s'il est vrai que la photographie - notamment dans le contexte de la presse et du photojournalisme - a fait l'objet d'un contrôle politique, de censure et de processus de sélection qui ont conduit certains aspects du conflit à ne pas être mis en images ou certaines images, même si elles avaient été réalisées, à ne pas connaître de diffusion. L'enquête menée dans le cadre de cette thèse auprès des photographes et des acteurs qui ont participé à produire et à diffuser des images (photographiques et filmiques) tout au long du conflit, et celle menée autour de certains fonds d'archives, bouscule quelque peu ce poncif. D'autant plus que cette idée fait l'objet de vives critiques parmi celles et ceux qui ont vécu et couvert le conflit. Posée autrement, la question des images manquantes concernerait non seulement les images qui n'ont pas été produites, mais les " modalités" par lesquelles certaines images "manquent ou viennent à manquer ", selon la formulation du philosophe Dork Buzanyan ${ }^{31}$, aussi bien pendant les années 1990 qu'aujourd'hui. C'est la réflexion que j'ai tenté d'amorcer à travers cet article, en décrivant, d'une part, le contexte économique, politique et les conditions matérielles de production, de diffusion et de conservation des photographies du conflit; d'autre part, en présentant une ébauche de cartographie des archives qui m'ont été ouvertes, des pratiques et des interactions entre les différents acteurs qui les détiennent et les manipulent au fil des années. Toutefois, il semble qu'une grande partie des images de ce conflit soient davantage latentes que manquantes, puisque leur résurgence dans le présent dépend de facteurs, d'événements et d'acteurs pouvant aussi bien provoquer, catalyser leur réapparition, que les amener à être reléguées dans une forme d'oubli, d'état latent. La question de la circulation et de la diffusion de ces images se pose en particulier dans le contexte de l'après 22 février 2019 - date symbolique du commencement du soulèvement populaire en cours depuis plus d'un an en Algérie dans lequel la période de la "décennie " (el 'achriya, les années 1990) fait l'objet de débats et de remises en question, plus visibles qu'auparavant dans l'espace public, notamment à travers les slogans et les chants, mais aussi les affiches et les photographies (par exemple, les portraits des disparus brandis par leurs proches dans les manifestations). 


\section{BIBLIOGRAPHIE}

ABOUT, Ilsen, CHÉROUX, Clément, « L'histoire par la photographie », Études photographiques, $n^{\circ} 10$, novembre 2001. [URL : http://journals.openedition.org/etudesphotographiques/261]. APPADURAI, Arjun, The Social Life of Things: Commodities in Cultural Perspective, Cambridge University Press, 1988.

ARANDA, Mauricio, SIMONPOLI, Nicolas, « Aller aux archives, entrer sur le terrain ? Sur les conditions sociales d'enquêtes en "terrain archivistique" ", Genèses, vol. 112, n 3, 2018, p. 123-139.

BECKER, Howard, Les Mondes de l'art, Paris, Champs, 2010 [1982]

BOURAS, Ammar, 1990-1995. Algérie, chronique photographique, Alger, Barzakh, 2018.

CHAREF, Abed, Algérie. Le grand dérapage, La Tour-d'Aigues, Éditions de l'Aube, 1994.

сноміNот, Marie, « Guerre des images, guerre sans image ? Pratiques et usages de la photographie pendant la guerre d'indépendance algérienne (1954-1962) », thèse de doctorat en histoire,

Paris VIII - Saint-Denis, 2008.

GROSSI, Valentina, «Produire l'actualité des images. Le travail d'assemblage dans les bureaux de

l'Agence France-Presse », Réseaux, n² 212, nº 6, 11 décembre 2018, p. 179-206.

KENNEDY, Liam, PATRICK, Caitlin, The Violence of the Image: Photography and International Conflict,

I.B.Tauris, 2014.

KHELASSI, Noureddine, « Rôle et marge de manœuvre de la presse algérienne », Esprit, $n^{\circ} 248$ (12), 1998, p. 225-235.

коРYTOFF, Igor, « The cultural biography of things: commoditization as process », The Social Life of Things, Arjun Appadurai (éd.), p. 64-92, Cambridge, Cambridge University Press, 1986.

LEBLANC, Audrey, L'Image de Mai 68 : du journalisme à l'histoire, thèse de doctorat en histoire, EHESS, 2015.

MARITNEZ, Luis, La Guerre civile en Algérie, Paris, Karthala, 1998.

MOUfFoK, Ghania, Ettre journaliste en Algérie, 1988-1995, Paris, Reporters sans Frontières, 1996.

MUNDY, Jacob, Imaginative Geographies of Algerian Violence: Conflict Science, Conflict Management, Antipolitics, Stanford University Press, 2015.

SEMIANE, Sid-Ahmed, Au refuge des balles perdues, Paris, La Découverte, 2005.

STORA, Benjamin, La guerre invisible: Algérie, années 1990, Paris, Presses de Sciences Po, 2001.

VON GRAFFENRIED, Michael, Algérie. Photographies d'une guerre sans images, Paris, éditions Hazan, 1998. ZAATARI, Akram, in conversation with DOWNEY, Anthony, « Photography as apparatus ", Ibraaz, 006, Janvier 2014 [URL : http://www.ibraaz.org/interviews/113]

ZABUNYAN, Dork, «Les images manquantes, ouverture », Les Images manquantes, Les Carnets du Bal, Paris, Le Bal, 2012.

\section{NOTES}

1. Traduction personnelle depuis le dardja (parler algérien) d'un extrait d'entretien avec G., octobre 2018: "L'archive, kayna, bessah machi palpable. Ma nendjemch na'tilek l'accès, ma nendjmech nwerihomlek. " Exceptionnellement, les mots en italique dans cet extrait sont prononcés en français. Dans le reste du texte et dans les notes de bas de page, les mots en italique sont en arabe ou en dardja. Toutes les traductions sont de l'auteure.

2. Dans les entretiens, les expressions employées pour désigner cette période sont davantage celles de "terrorisme» ou "irhab» en dardja, «waqt el irhab» (temps du terrorisme), «la décennie » (el 'achriya) ou "décennie noire », que celui de "guerre » ou "guerre civile », bien 
moins souvent mobilisés. L'État algérien emploie quant à lui l'expression de «tragédie nationale » dans les textes officiels, évitant ainsi toute politisation de la question. Si la notion de " guerre civile " permet en effet d'échapper à une lecture partisane du conflit et de dépasser le discours des protagonistes (Martinez, 1998), elle reste largement rejetée en Algérie. N'ayant pas l'espace pour développer ce point, j'utiliserai ici les expressions employées par mes interlocuteurs pour désigner cette période, ainsi que les mots « conflit » et " guerre », plutôt que « guerre civile».

3. Il s'agit ici de préciser quels mots sont employés par mes interlocuteurs dans nos échanges pour désigner ces photographies aujourd'hui. Le terme " archives ", s'il renvoie à un vaste champ théorique, particulièrement en Europe (Arlette Farge, Jacques Derrida, notamment), est avant tout mobilisé dans cet article en tant que catégorie " native ", employé par les acteurs.

4. J'ai fait le choix d'anonymiser les noms des personnes avec lesquelles je travaille tant que mon enquête de terrain est en cours et de ne dévoiler que les noms des acteurs dont la production photographique des années 1990 fait déjà l'objet d'une médiatisation aujourd'hui, à l'exemple du travail d'Ammar Bouras depuis la parution de son livre de photographies: Ammar Bouras, 1990-1995. Algérie, chronique photographique, Alger, Barzakh, 2018.

5. Ilsen About, Clément Chéroux, «L'histoire par la photographie », Études photographiques, $\mathrm{n}^{\circ} 10$, novembre 2001. [URL : http://journals.openedition.org/etudesphotographiques/261].

6. Ibid.

7. Une telle démarche rejoint, par exemple, les propositions des politologues Mauricio Aranda et Nicolas Simonpoli à propos de la possibilité d'appréhender les archives comme un terrain à part entière. Mauricio Aranda, Nicolas Simonpoli, «Aller aux archives, entrer sur le terrain ? Sur les conditions sociales d'enquêtes en "terrain archivistique" ", Genèses, vol. 112, n 3, 2018, p. 123-139 [URL : https://www.cairn.info/revue-geneses-2018-3-page-123.htm?contenu=article].

8. Arjun Appadurai, The Social Life of Things: Commodities in Cultural Perspective, Cambridge University Press, 1988.

9. Akram Zaatari, in conversation with Anthony Downey, "Photography as apparatus ", Ibraaz, 006, janvier 2014 [URL : http://www.ibraaz.org/interviews/113].

10. Concernant le contexte politique de la création de la presse privée, se référer notamment aux écrits suivants : Abed Charef, Algérie. Le grand dérapage, La Tour-d'Aigues, Éditions de l'Aube, 1994, p. 11-14; Noureddine Khelassi, "Rôle et marge de manœuvre de la presse algérienne », Esprit, n 248 (12), 1998, p. 225-235 ; Ghania Mouffok, Être journaliste en Algérie, 1988-1995, Paris, Reporters sans Frontières, 1996.

11. Parti islamiste majoritaire en Algérie à cette période.

12. Sid-Ahmed Semiane, Au refuge des balles perdues, Paris, La Découverte, 2005.

13. Liberté et El-Watan sont des journaux privés francophones. Horizons est un journal public, dont une partie de l'équipe fondatrice est issue d'El Moudjahid, organe du FLN.

14. Valentina Grossi, « Produire l'actualité des images. Le travail d'assemblage dans les bureaux de l'Agence France-Presse ", Réseaux n 212, nº 6, 11 décembre 2018, p. 179-206.

15. La plupart des photographes que j'ai rencontrés affirment n'avoir remis aux rédactions que des tirages et avoir conservé leurs négatifs.

16. «Patriotes » est le mot désignant les membres d'une catégorie de milices civiles constituées pendant les années 1990 et armées par l'État algérien.

17. Entretien avec O., juillet 2019.

18. Entretien avec M., avril 2017.

19. La loi dite de la Concorde civile est promulguée par référendum en septembre 1999. Elle est suivie de la Charte pour la réconciliation nationale et la paix, promulguée suite à un référendum en août 2005 et mise en œuvre à partir de février 2006. Ces deux lois restent controversées en Algérie. 
20. Par exemple, des artistes comme Sofiane Zouggar, Ammar Bouras, Katia Kameli et des réalisateurs comme Djamel Kerkar ont, chacun à sa façon, mobilisé des photographies et des vidéos du conflit des années 1990 dans leurs récentes productions et leurs recherches.

21. Entretien avec le photographe, novembre 2018.

22. Ces logements sécuritaires ont été aménagés pour les travailleurs de la presse à partir de 1993, alors qu'une série d'assassinats ciblant les journalistes avait commencé.

23. Par exemple : en 2003, "L'Algérie, les faits et les effets. 12 ans de photojournalisme ", dans le cadre de l'événement «Algérie, un autre regard» organisé en partenariat avec la Fnac dans plusieurs villes de France; en 2005, «Panoramas de la photographie algérienne », organisé avec la Wilaya de Tipaza en Algérie dans plusieurs villes; en 2012, le Festival de la photographie algérienne (FesPA) à Tlemcen, Algérie.

24. Citons, par exemple, le site de Getty images, qui donne accès à une partie des archives de l'agence Sygma, aujourd'hui inactive, celui de l'agence Gamma, ou encore celui de la fondation Magnum, qui contient quelques photographies du conflit des années 1990, notamment celles de Abbas et de Paolo Pellegrin. Des photographies sont aussi publiées sur certains sites d'informations et blogs, notamment celles de Michael Von Graffenried et des photographes d'agences de presse et d'agences photographiques précédemment citées.

25. L'image latente en photographie désigne l'image « en devenir » présente sur un film exposé mais pas encore développé. C'est le sentiment d'être confrontée à des images latentes, plutôt qu'à des images absentes ou manquantes, tout au long de ce travail de recherche, qui m'a amenée à emprunter ce terme, à l'origine technique et lié à la chimie de la photographie, pour désigner un ensemble d'images qui existent matériellement mais ne sont pas visibles et dont on ignore si leur trajectoire les amènera ou non à ressurgir dans le présent.

26. Traduction personnelle : « [...] had the studio been an active studio today, had the economy of photography still been active today, Madani would not let me take one negative out of his archive. [...] So the death of his economy made my project possible... », dans Akram Zaatari, Anthony Downey, art. cit., p. 5-6.

27. Il existe une dizaine de livres de photographies portant sur la période des années 1990 en Algérie. Parmi eux, les livres de photographies de presse ou de photographies documentaires sont ceux de Akram Belkaïd et Jean-Pierre Peyroulou, L'Algérie en guerre civile, Paris, CalmannLévy, 2002, de Chérif Benyoucef, Une saison en enfer, Parangon, 2003, et les livres de Michael von Graffenried, publiés dans plusieurs pays (France, États-Unis, Allemagne, Algérie).

28. Le livre a paru à la fin de l'année 2018 chez les éditions Barzakh, à Alger, mais n'a été commercialisé qu'au début de l'année 2019.

29. Ces notions sont développées dans l'entretien entre Akram Zaatari et Anthony Downey, art. cit.

30. Par exemple, Michael von Graffenried, Algérie. Photographies d'une guerre sans images, Paris, éditions Hazan, 1998 ; Benjamin Stora, La Guerre invisible: Algérie, années 1990, Paris, Presses de Sciences Po, 2001.

31. Dork Zabunyan, «Les images manquantes, ouverture », Les Images manquantes, Les Carnets du Bal, Paris, Le Bal, 2012. 


\section{RÉSUMÉS}

L'article propose une réflexion autour des archives photographiques du conflit des années 1990 en Algérie. En s'appuyant sur une ethnographie réalisée dans le présent, il retrace la trajectoire de photographies produites par des photographes de presse dans les années 1990, et questionne la place qu'elles occupent aujourd'hui, à la fois en tant qu'objets matériels et images. Interrogeant la notion d'archive(s) dans le contexte algérien, l'article propose une description des contextes et des pratiques qui ont participé à façonner ces photographies à travers le temps, en s'intéressant aux images dans les rapports que les acteurs entretiennent avec elles.

The article examines the photographic archives of the 1990s conflict in Algeria. Based on an ethnography carried out in the present, it traces the trajectory of photographs produced by press photographers in the 1990s, and questions the place they occupy today, both as material objects and images. Questioning the notion of archive(s) in the Algerian context, the article attempts to describe the contexts and practices that have helped shape these photographs over time, focusing on the images and their relationships to the actors.

\section{INDEX}

Index chronologique : 1990

Index géographique : Algérie

Mots-clés : Algérie, années 1990, guerre, conflit, violence, photographie, photojournalisme, archives, presse, passé dans le présent

Keywords : Algeria, 1990s, war, conflict, violence, photography, photojournalism, archives, press, past in the present

\section{AUTEUR}

\section{AWEL HAOUATI}

Awel haouati est doctorante à l'EHESS (Paris, Anthropologie sociale et ethnologie). Ses recherches portent sur les pratiques et les usages politiques de la photographie dans le contexte du conflit des années 1990 en Algérie. Elle s'intéresse aux trajectoires de photographes de presse qui ont travaillé en Algérie à cette période. 